\title{
Classification of dry-cured hams according to the maturation time using near infrared spectra and artificial neural networks
}

\author{
M. Prevolnik a, D. Andronikov ${ }^{\text {b }}$, B. Žlender ${ }^{\text {b }}$, M. Font-i-Furnols ${ }^{c}$, M. Novič ${ }^{\mathrm{d}}$, \\ D. Škorjanc ${ }^{a}$, M. Čandek-Potokar ${ }^{\mathrm{e}, *}$ \\ a University of Maribor, Faculty of Agriculture and Life Sciences, Pivola 10, 2311 Hoče, Slovenia \\ b University of Ljubljana, Biotechnical Faculty, Jamnikarjeva 101, 1000 Ljubljana, Slovenia \\ C IRTA - Institute of Agri-Food Research and Technology, Finca Camps i Armet, 17121 Monells, Girona, Spain \\ d National Institute for Chemistry, Hajdrihova ulica 19, 1000 Ljubljana, Slovenia \\ e Agricultural Institute of Slovenia, Hacquetova ulica 17, 1000 Ljubljana, Slovenia
}

\section{A R T I C L E I N F O}

\section{Article history}

Received 7 November 2012

Received in revised form 24 May 2013

Accepted 10 June 2013

Available online 15 June 2013

Keywords:

Dry-cured ham

Classification

Near infrared spectroscopy

Artificial neural networks

\begin{abstract}
A B S T R A C T
An attempt to classify dry-cured hams according to the maturation time on the basis of near infrared (NIR) spectra was studied. The study comprised 128 samples of biceps femoris (BF) muscle from dry-cured hams matured for $10(\mathrm{n}=32), 12(\mathrm{n}=32), 14(\mathrm{n}=32)$ or 16 months $(\mathrm{n}=32)$. Samples were minced and scanned in the wavelength range from 400 to $2500 \mathrm{~nm}$ using spectrometer NIR System model 6500 (Silver Spring, MD, USA). Spectral data were used for i) splitting of samples into the training and test set using $2 D$ Kohonen artificial neural networks (ANN) and for ii) construction of classification models using counterpropagation ANN (CP-ANN). Different models were tested, and the one selected was based on the lowest percentage of misclassified test samples (external validation). Overall correctness of the classification was 79.7\%, which demonstrates practical relevance of using NIR spectroscopy and ANN for dry-cured ham processing control.
\end{abstract}

(ㄷ) 2013 Elsevier Ltd. All rights reserved.

\section{Introduction}

Dry-cured ham "Kraški pršut" is a traditional Slovenian meat product protected with EU designation of geographical indication (Commission implementing regulation, 2012). This protection implies that certain consortium rules should be respected in regard to raw material, processing losses, chemical and sensory properties. Processing of dry-cured ham "Kraški pršut" consists of dry salting, absence of smoking and long maturation period. Regarding the latter, the consortium rules require a minimum maturation period of 12 months. During seasoning, proteins and lipids undergo intense proteolysis and lipolysis processes and these changes affect the flavour and texture of dry-cured ham, resulting in sensorial quality appreciated by consumers. Chemical and sensory changes occurring through the process are strongly dependent on the duration of the ripening process which has been demonstrated in different dry-cured meat products (Benedini, Parolari, Toscani, \& Virgili, 2012; Buscailhon \& Monin, 1994; Toldra \& Flores, 1998 and Virgili, Saccani, Gabba, Tanzi, \& Soresi Bordini, 2007). From the practical point of view it would be interesting to develop cheap and rapid method capable of detecting ham ripening stage. In the case of "Kraški pršut" such approach would be useful for verification purposes (e.g. to detect

\footnotetext{
* Corresponding author. Tel.: + 38612805 124; fax: + 38612805255 . E-mail address: meta.candek-potokar@kis.si (M. Čandek-Potokar).
}

if the requirement of 12 months of maturation was respected) or to recognize longer maturation associated with higher quality label. Near infrared (NIR) spectroscopy is one of the techniques which have the potential for such purposes. Its usefulness has already been proven for the prediction of chemical and physical characteristics of meat and meat products and for various classification purposes (for review see Prevolnik, Čandek-Potokar, \& Škorjanc, 2004 and Prieto, Roehe, Lavín, Batten, \& Andrés, 2009). NIR spectral information demands multivariate data analysis due to its complexity (Pérez-Marín, Garrido-Varo, \& Guerrero, 2007). Artificial intelligent methods are often applied for the classification since their primary target is to distinguish objects or groups or populations. Their advantages are in ability to handle with non-linear data, highly correlated variables and potential for identification of problems or classification (Cartwright, 2008). Artificial neural networks (ANN) were lately tested for many problems in meat production and technology such as carcass classification, quality control of raw material, meat processing, meat spoilage or freshness and shelf-life evaluation, detecting off-flavours, authenticity assessment, etc. (for review see Prevolnik, Škorjanc, Čandek-Potokar, \& Novič, 2011). To our knowledge there is no literature data on the prediction of maturation time in any of dry-cured meat products based on NIR spectral information. Therefore the present study aimed to test if dry-cured hams can be classified into different maturation classes ( 10 , 12,14 and 16 months) on the basis of spectral information by means of ANN. 


\section{Materials and methods}

\subsection{Ham processing and sampling}

The study comprised 128 dry-cured hams taken from regular production in three commercial ham processing facilities (members of consortium for Kraški pršut). The production of hams was carried out respecting the rules of consortium for Kraški pršut. In short, the hams from commercial crossbred pigs were trimmed into a prescribed shape and put to salting for $2-3$ weeks at $2-4{ }^{\circ} \mathrm{C}$. According to the rules only sea salt is allowed as a conservation additive. After the salting, the hams were washed and left to rest in controlled atmosphere (at $4-6{ }^{\circ} \mathrm{C}$ and $70-85 \%$ relative humidity) for 10 weeks. Following the resting period the hams were dried $\left(14-20{ }^{\circ} \mathrm{C}\right.$ and $\left.60-80 \% \mathrm{RH}\right)$ until the required weight loss had been attained. Thereafter the open surface of the hams was coated with a mixture of pork leaf fat, rice flower and spices (to permit ripening while preventing further desiccation) and left to ripen. Hams were sampled after 10 $(\mathrm{n}=32), 12(\mathrm{n}=32), 14(\mathrm{n}=32)$ and 16 months $(\mathrm{n}=32)$ of seasoning in all three dry-cured ham processing facilities. Thereafter the hams were boned and sampled from the central part of the dry-cured hams containing biceps femoris and semimembranosus muscles according to Škrlep et al. (2012).

\subsection{NIR spectra acquisition}

Biceps femoris muscle samples ( $2 \mathrm{~cm}$ thick slice) were trimmed of superficial fat tissue, cut in small pieces, frozen in liquid nitrogen and grinded to fine dust using a laboratory mill (IKA M120, IKA Werke, Staufen, Germany). Homogenized ham samples (app. $50 \mathrm{~g}$ ) were put in rectangular quartz cup $\left(47 \times 57 \mathrm{~mm}^{2}\right)$ about $3 \mathrm{~mm}$ thick, covered by paper disc and placed directly in NIRS apparatus NIR System model 6500 (Silver Spring, MD, USA). For each sample one scanning was performed in a wavelength range from 400 to $2500 \mathrm{~nm}$. Absorbance data were collected every $2 \mathrm{~nm}$ as $\log 1 / R$, where $R$ represents the reflectance.

\subsection{Dry-cured ham chemical composition}

Several chemical constituents (moisture, intramuscular fat, protein, non-protein nitrogen, salt content, the percentage of salt per dry mater and proteolysis index) of dry-cured ham muscle biceps femoris were determined by means of internal NIR spectroscopy calibration models published in Prevolnik, Škrlep, Janeš, Velikonja-Bolta, Škorjanc \& Čandek-Potokar (2011). Data are presented in Table 1.

\subsection{Chemometric analysis}

Chemometric analysis was performed using ANN software developed at the National Institute for Chemistry (Ljubljana, Slovenia), written in FORTRAN for IBM-compatible PCs and a Windows operating system. In the present study, unsupervised Kohonen ANN and supervised counter-propagation (CP) ANN were applied. Although both types of ANN are comprehensively described in the literature (Dayhof, 1990; Hecht-Nielsen, 1987; Zupan, 1994 and Zupan, Novič, \& Ruisanchez, 1997), a short explanation of them is given in the next paragraphs.

In the case of unsupervised learning strategy, only the description of objects are needed, i.e. the independent variables for the input vectors. The properties are not given, so the map obtained shows only the relationship between the independent variables of the objects, regardless of their property that may be known, but is not represented in object vectors. The main goal of Kohonen ANN is to project or map objects from m-dimensional into 2-dimensional space on the basis of input data (similarity among objects). Thus Kohonen ANN is most frequently applied for visualization and clustering purposes (Zupan, 1994).
Table 1

Chemical composition of dry-cured hams (means and standard deviations).

\begin{tabular}{lllll}
\hline & \multicolumn{4}{l}{ Maturation time (months) } \\
\cline { 2 - 5 } Constituent $^{\mathrm{a}}$ & 10 & 12 & 14 & 16 \\
\hline${ }^{1}$ Moisture, g/kg & $592 \pm 16.1$ & $567 \pm 21.8$ & $582 \pm 15.1$ & $564 \pm 22.8$ \\
${ }^{2}$ Salt, g/kg & $70.3 \pm 10.38$ & $76.7 \pm 10.18$ & $77.0 \pm 8.30$ & $78.1 \pm 8.38$ \\
${ }^{3}$ Salt per dry mater, \% & $17.2 \pm 2.03$ & $17.7 \pm 2.11$ & $18.4 \pm 1.67$ & $17.9 \pm 1.60$ \\
${ }^{4}$ Protein, g/kg & $27.8 \pm 1.07$ & $29.5 \pm 1.64$ & $29.6 \pm 1.07$ & $30.1 \pm 1.58$ \\
${ }^{5}$ Non-protein nitrogen, & $11.6 \pm 0.51$ & $11.8 \pm 1.50$ & $12.7 \pm 1.00$ & $12.6 \pm 1.45$ \\
$\quad$ g/kg & & & & \\
${ }^{6}$ Proteolysis index, \% & $27.1 \pm 1.05$ & $26.1 \pm 3.14$ & $27.8 \pm 1.75$ & $27.2 \pm 2.55$ \\
${ }^{7}$ Intramuscular fat, g/kg & $42 \pm 7.3$ & $43 \pm 20.3$ & $30 \pm 9.0$ & $40 \pm 13.7$ \\
\hline
\end{tabular}

a Assessed with NIR spectroscopy using internal calibration models (Prevolnik, Škrlep, et al., 2011) with the following chemometric parameters:

${ }^{1} \mathrm{n}=131, \mathrm{se}_{\mathrm{c}}=4.5, \mathrm{R}_{\mathrm{c}}^{2}=0.89, \mathrm{se}_{\mathrm{cv}}=5.0, \mathrm{R}_{\mathrm{cv}}^{2}=0.86$

${ }^{2} \mathrm{n}=130, \mathrm{se}_{\mathrm{c}}=1.23, \mathrm{R}^{2}{ }_{\mathrm{c}}=0.97, \mathrm{se}_{\mathrm{cv}}=1.44, \mathrm{R}_{\mathrm{cv}}^{2}=0.96$

${ }^{3} \mathrm{n}=129, \mathrm{se}_{\mathrm{c}}=0.42, \mathrm{R}_{\mathrm{c}}^{2}=0.92, \mathrm{se}_{\mathrm{cv}}=0.46, \mathrm{R}_{\mathrm{cv}}^{2}=0.90$

${ }^{4} \mathrm{n}=131, \mathrm{se}_{\mathrm{c}}=0.49, \mathrm{R}_{\mathrm{c}}^{2}=0.81, \mathrm{se}_{\mathrm{cv}}=0.55, \mathrm{R}_{\mathrm{cv}}^{2}=0.77$

${ }^{5} \mathrm{n}=131, \mathrm{se}_{\mathrm{c}}=0.28, \mathrm{R}_{\mathrm{c}}^{2}=0.88, \mathrm{se}_{\mathrm{cv}}=0.36, \mathrm{R}_{\mathrm{cv}}^{2}=0.80$

${ }^{6} \mathrm{n}=130, \mathrm{se}_{\mathrm{c}}=0.67, \mathrm{R}^{2}{ }_{\mathrm{c}}=0.78, \mathrm{se}_{\mathrm{cv}}=0.83, \mathrm{R}_{\mathrm{cv}}^{2}=0.67$

${ }^{7} \mathrm{n}=128, \mathrm{se}_{\mathrm{c}}=3.00, \mathrm{R}_{\mathrm{c}}^{2}=0.88, \mathrm{se}_{\mathrm{cv}}=3.16, \mathrm{R}_{\mathrm{cv}}{ }=0.87$.

The CP-ANN is based on two-steps learning procedure. The first step corresponds to the mapping of objects in the input layer (also called Kohonen layer) and is identical to the Kohonen learning procedure. The second step of the learning is supervised, which means that for the learning procedure the response or target value is required for each input. This input-target pairs are the input to the neural network, which is after being trained for certain amounts of epochs, capable of the prediction of the unknown samples. Every object excites one single neuron. The algorithm modifies the weight of the neuron with the weights most similar to the input signal and smoothes the map by making modulated changes to neurons in a defined "neighbourhood" of that one. These corrections of weights are made around the neuron position in the Kohonen and output layer (Zupan, 1994).

\subsection{Selection of sample sets}

Composition of the training and the test sets should guarantee that these sets are scattered over the similar descriptor spaces and the training set is a representative set of the whole data set and Kohonen ANN are able to select a representative training set and a test set similar to it (Gramatica, Pilutti, \& Papa, 2004).

For chemometric analysis, absorbance data collected every $2 \mathrm{~nm}$ ( $\mathrm{n}=1032$ ) were compressed into 262 data points by averaging four sequential original measurements. Each sample is represented as a combination of 262 input variables or descriptors. For this unsupervised step only input variables are needed. The distribution of samples $(\mathrm{n}=128)$ in the top map of the Kohonen ANN was used to divide samples into the training and test set. Splitting of the samples was performed for each of four maturity classes separately (10, 12,14 and 16 months). Samples of certain maturity class $(n=32)$ were trained using $4 \times 4$ Kohonen net for 100 epochs. After the training, each sample obtained the location in the Kohonen map, i.e. in a rectangular grid of neurons. Among the samples sited on the same neuron, the one with the shortest distance to the neuron was selected for the training set, while the remaining sample(s) was (were) placed in the test set. For each maturity class 16 samples were selected for training set and the others were placed into the test set. The final training set ( $\mathrm{n}=64$ ) was used for the development of calibration models and test set $(n=64)$ for independent external validation of models.

\subsection{Development and validation of models}

In the present study, the CP-ANN was employed as a classification model. Different models were prepared varying the net size, i.e. the 
number of neurons in $x$ and $y$ direction $(8 \times 8,10 \times 10$ and $12 \times 12)$ and number of epochs (10, 30, 50, 70, 100, 150, 200 and 300), while the other net parameters remained constant (no toroid boundary conditions, triangular type of neighbourhood correction, minimal and maximal learning rates 0.01 and 0.5 , respectively). In this step of analysis, besides the input variables (262 spectral data points), the target or a)

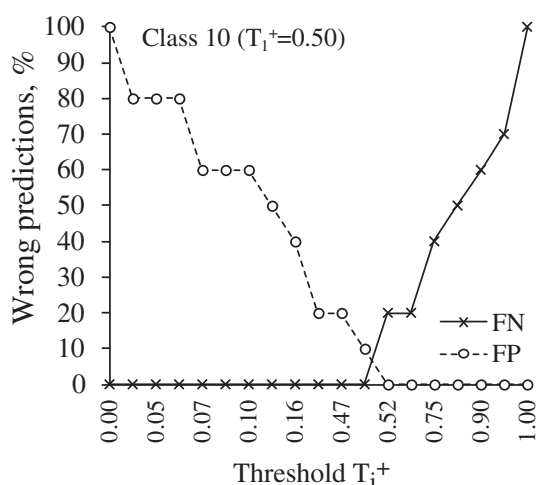

b)

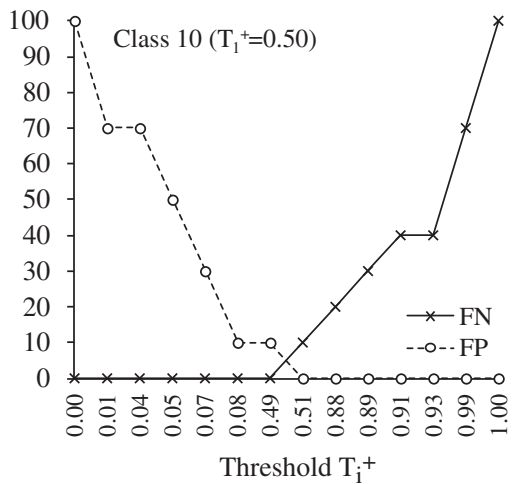

c)

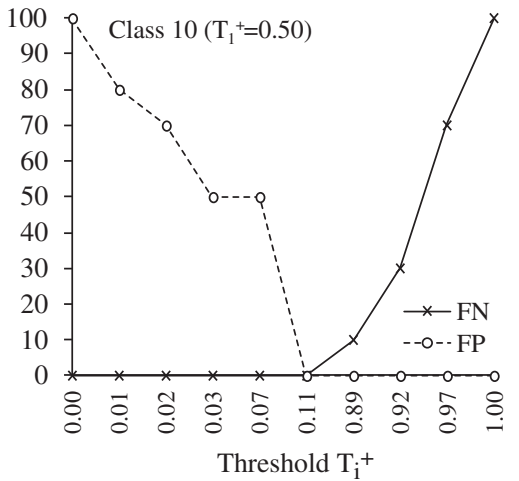

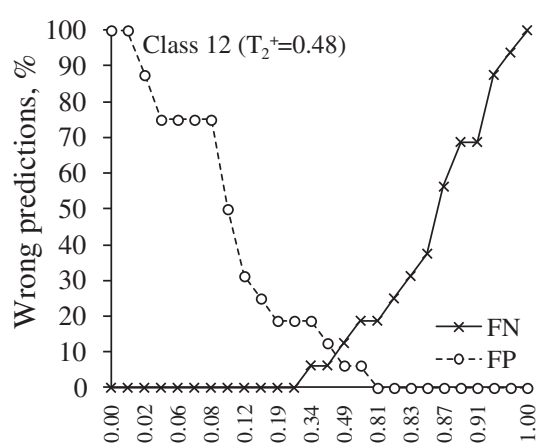

Threshold $\mathrm{T}_{\mathrm{i}}^{+}$

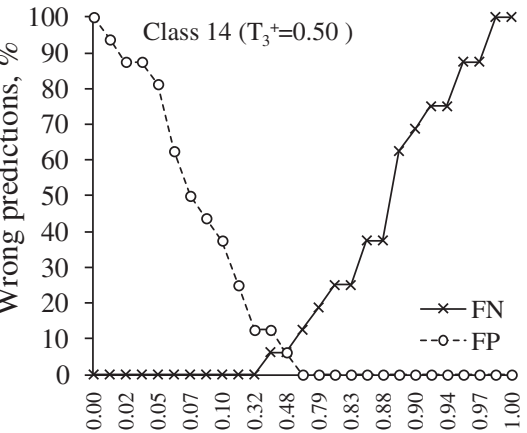

Threshold $\mathrm{T}_{\mathrm{i}}^{+}$

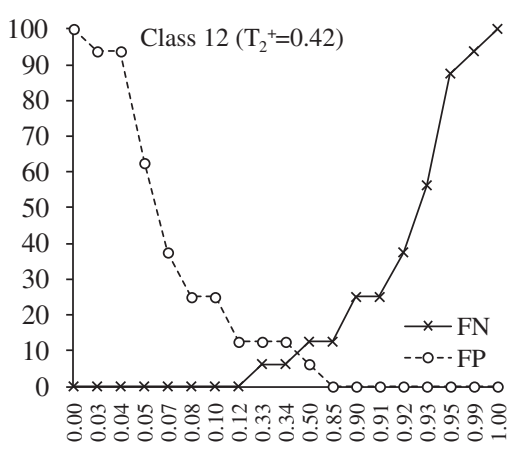

Threshold $\mathrm{T}_{\mathrm{i}}^{+}$

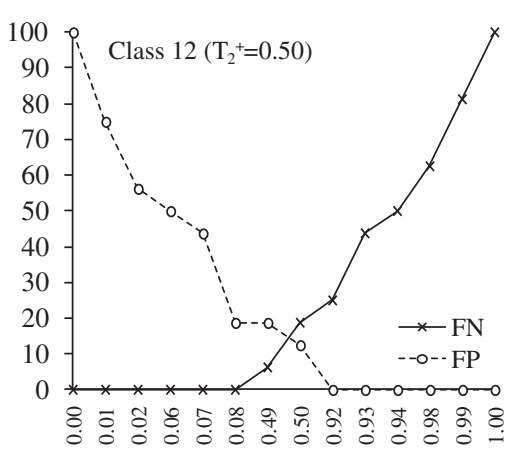

Threshold $\mathrm{T}_{\mathrm{i}}^{+}$

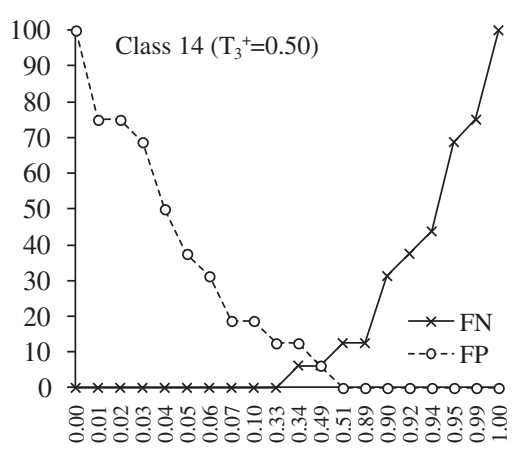

Threshold $\mathrm{T}_{\mathrm{i}}{ }^{+}$

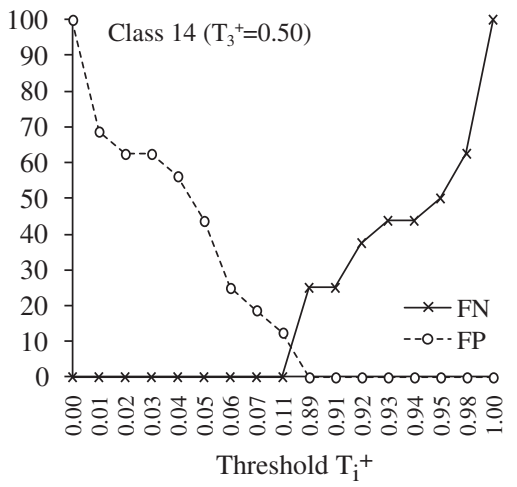

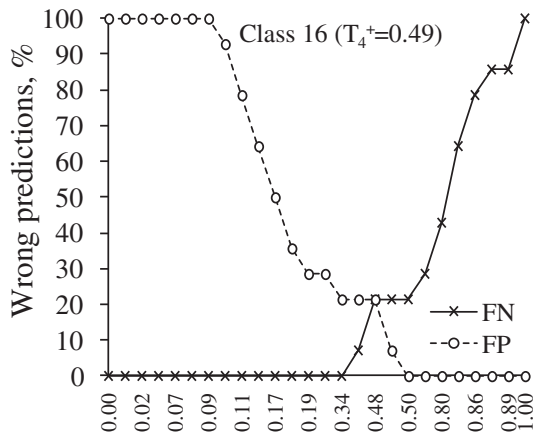

Threshold $\mathrm{T}_{i}^{+}$

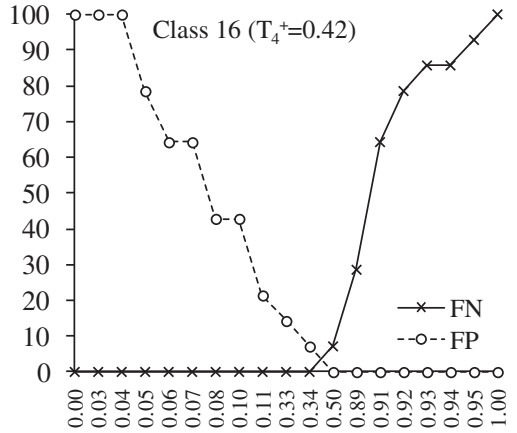

Threshold $\mathrm{T}_{i}^{+}$

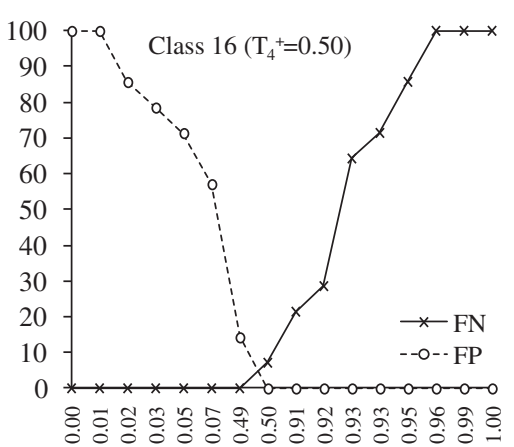

Threshold $\mathrm{T}_{i}^{+}$

$\mathrm{T}_{\mathrm{i}}^{+}$- treshold value $\quad \mathrm{FP}-$ False positive; FN - False negative

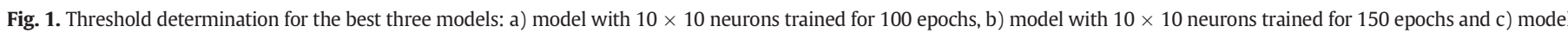
with $12 \times 12$ neurons trained for 200 epochs. 
output variables were also needed. The target variable i.e. maturity class with four levels (10, 12, 14 and 16 months of ripening) was given as a combination of four discrete values (ones and zeros) for each of the four levels for each sample. After the training, for each sample, a combination of four real numbers between 0.0 and 1.0 (denoting probability to belong to a respective class) was obtained. To convert this information into a "prediction of maturation class" the real numbers had to be transformed first to discrete values (ones and zeros). For that, it was necessary to determine the threshold values for each of the four classes below which all the predictions were negative and denoted by a zero (which means that certain sample does not belong to the certain class), while the predictions above the threshold were positive and denoted by one. The threshold values were determined for all developed models in order to be able to obtain class predictions when testing the predictive ability on 64 independent samples (external validation). Classification performance was evaluated through the number of correctly classified samples in the independent set.

\section{Results and discussion}

\subsection{Determination of threshold values}

Classification of samples according to maturation time was performed using a nonlinear modelling method, i.e. CP-ANN. Prior to the interpretation of the results some presentation of the chemometric analysis should be given, particularly of the threshold values determination. When predicting classes using CP-ANN, the result of prediction is real numbers for every class, which have to be converted back to the discrete values. This is a demanding task related to the classification which requires a determination of thresholds above which the prediction of a certain class is positive (confirmative) and below which the prediction is negative (rejecting). In this part of the chemometric analysis we followed the procedure described in Roncaglioni, Novič, Vračko, and Benfenati (2004). Examples of threshold determination for three constructed models are presented in Fig. 1. These thresholds had to be determined separately for individual (four) classes. For each constructed model different possible thresholds between 0.00 and 1.00 were tested. They were chosen with regard to the values obtained from the output layer of individual models. Below the tested threshold all predictions are denoted by a zero, which means that the ham sample does not belong to certain maturity class, while the predictions above the threshold are denoted by one (the sample belongs to certain maturity class). If the tested threshold is close to zero the predictions of certain class for most of the samples from the training set would be confirmative (one). The samples from certain class would be correctly predicted, while the predictions for the rest of the samples would be so called false positives. On the other hand, if the tested thresholds are close to one, the majority of predictions for certain class would be rejected (zero). This would produce false negative predictions of the samples that actually belong to a particular class. Among several tested thresholds (for each class of each model) one was selected for further use (i.e. for validation of models) according to the number of correct/wrong class predictions of the samples from the training set. As can be seen from Fig. 1, the selected threshold was located where the sum of errors, of both false positive and false negative predictions, was the lowest. The thresholds for the best three models (net with $10 \times 10$ trained for 100 and 150 epochs and net with $12 \times 12$ trained for 200 epochs) were positioned between 0.42 and 0.50 (Fig. 1). Once the thresholds were determined, the models were validated by checking the class-predictions of test samples respecting selected thresholds.

\subsection{Classification results}

Altogether, 24 CP-ANN classification models were developed in the training step and further tested on 64 independent samples (training set) to assess their predictive ability. Validation results (Table 2) are presented as the number of hams that were correctly classified according to the ripening time. In Table 2 we can examine classification results when varying the net size $(8 \times 8,10 \times 10$ and $12 \times 12)$ and the number of epochs (10, 30, 50, 70, 100, 150, 200 and 300). Regarding the net size, a bit lower correctness was observed in the case of a smaller net (the net with eight neurons in $x$ and $y$ direction) although the differences were not substantial. A somewhat bigger variability was caused when varying the number of epochs used to train the net. We can observe inferior results in

Table 2

Presentation of CP-ANN models for classification of hams according to the maturation time based on spectral data.

\begin{tabular}{|c|c|c|c|c|c|c|}
\hline \multirow[b]{3}{*}{ Net size } & \multirow[b]{3}{*}{ No. of epochs } & \multicolumn{4}{|c|}{ Correctly classified samples ${ }^{\mathrm{a}}$} & \multirow{3}{*}{$\begin{array}{l}\text { Overall correctness } \\
\text { of classification } \\
(\mathrm{n}=64)\end{array}$} \\
\hline & & \multicolumn{4}{|c|}{ Maturation time (months) } & \\
\hline & & $10(\mathrm{n}=16)$ & $12(\mathrm{n}=16)$ & $14(\mathrm{n}=16)$ & $16(\mathrm{n}=16)$ & \\
\hline $8 \times 8$ & 10 & 11 & 8 & 11 & 8 & 38 \\
\hline $8 \times 8$ & 30 & 13 & 10 & 11 & 8 & 42 \\
\hline $8 \times 8$ & 50 & 13 & 10 & 11 & 8 & 42 \\
\hline $8 \times 8$ & 70 & 11 & 12 & 12 & 9 & 44 \\
\hline $8 \times 8$ & 100 & 13 & 10 & 13 & 8 & 44 \\
\hline $8 \times 8$ & 150 & 13 & 11 & 13 & 5 & 42 \\
\hline $8 \times 8$ & 200 & 11 & 11 & 13 & 10 & 45 \\
\hline $8 \times 8$ & 300 & 11 & 13 & 11 & 8 & 43 \\
\hline $10 \times 10$ & 10 & 11 & 8 & 13 & 6 & 38 \\
\hline $10 \times 10$ & 30 & 11 & 10 & 12 & 9 & 42 \\
\hline $10 \times 10$ & 50 & 13 & 11 & 9 & 10 & 43 \\
\hline $10 \times 10$ & 70 & 13 & 12 & 10 & 9 & 44 \\
\hline $10 \times 10$ & 100 & 12 & 13 & 14 & 10 & 49 \\
\hline $10 \times 10$ & 150 & 12 & 12 & 14 & 11 & 49 \\
\hline $10 \times 10$ & 200 & 12 & 11 & 9 & 8 & 40 \\
\hline $10 \times 10$ & 300 & 13 & 12 & 10 & 9 & 44 \\
\hline $12 \times 12$ & 10 & 10 & 8 & 12 & 6 & 36 \\
\hline $12 \times 12$ & 30 & 13 & 12 & 12 & 9 & 46 \\
\hline $12 \times 12$ & 50 & 12 & 12 & 10 & 11 & 45 \\
\hline $12 \times 12$ & 70 & 12 & 11 & 10 & 9 & 42 \\
\hline $12 \times 12$ & 100 & 14 & 12 & 12 & 8 & 46 \\
\hline $12 \times 12$ & 150 & 13 & 12 & 12 & 8 & 45 \\
\hline $12 \times 12$ & 200 & 14 & 11 & 14 & 12 & 51 \\
\hline $12 \times 12$ & 300 & 12 & 11 & 15 & 8 & 46 \\
\hline
\end{tabular}

\footnotetext{
a Independent test samples.
} 
the case of the smallest number of epochs. Higher number of epochs also produced poorer results compared to the models trained for 100 to 200 epochs, which is probably due to overfitting. The highest rate of correctness (i.e. 79.7\%) was obtained with $12 \times 12$ and 200 epochs. Models with $10 \times 10$ neurons trained for 100 or 150 epochs produced similar results (76.6\% of correctly classified test samples) and were thus only slightly behind the best one. When looking at the overall correctness these three models are comparable, however, differences in prediction of single classes can be observed among them.

Further analysis of the best three models included construction of misclassification tables (Fig. 2), which were obtained by the comparison of actual and predicted classes of test samples. In misclassification tables, correctly classified samples are presented as the diagonal elements, false positives as the upper triangle and false negative as the lower triangle. If a sample could not be classified into a single class, it was labelled as uncertain. In general, we can observe very good classification accuracy for 14 month matured hams for all three models. There was none or one misclassified and one or two uncertainly predicted samples. Regarding other maturity classes, up to four samples were wrongly and uncertainly classified. The 12 month matured hams were most accurately predicted by the first model $(10 \times 10,100$ epochs $)$, while we can observe the most successful prediction of 10 and 16 month matured hams using the last model $(12 \times 12,200$ epochs). Among these three models the best one was chosen on the basis of the largest number of correct predictions (sum of the diagonal elements), and the smallest number of false positive predictions. Respecting these criteria, the model with $12 \times 12$ neurons and 200 epochs proved the best performance for class predictions. The main weakness of this model is poorer accuracy to classify 12-months old hams which are on the other hand better predicted using the first one $(10 \times 10$, 100 epochs).

In Fig. 3, the predictions using the best model $(12 \times 12,200$ epochs) on the test set are presented in details. Besides the number of correct, wrong and uncertain cases the probabilities to predict individual class can be seen for all test samples. The probability of class prediction decreased with maturation time as demonstrated by higher probability rates for correctly classified hams in 10- and 12-months groups than in 14- and 16-months groups. It is also worth noting that 10 out of 64 hams were predicted with similar probability into two classes The observed decline in the probabilities as well as the correctness of the classification can be related to the chemical changes, in particular water content and activity, of which the intensity slows down in the course of the seasoning (from 10 to 16 months).

From the practical point of view, correct classification of hams according to the maturation time is important from two aspects. Firstly, a period of 12 months of maturation is required by the consortium for Kraški pršut and secondly, longer maturation (i.e. 16 months) is associated with additional quality label. With respect to the first aspect the worst situation that can happen is to classify a 10-month ham as 12 , 14 or 16-month one. This happened with two hams, no. 11 and no. 14 , being unjustifiably classified as 12 - and 16-months, respectively. It would also be undesirable to classify $12-, 14$ - or 16 -month hams as 10 -month hams because in this case a ham that fulfils the criteria of 12 -months of maturation would be declassified. However, such situation did not appear with our samples (only the sample no. 51 aged for 16 -months was close to that with a probability 0.475 for class 10 -months and 0.479 for 16 -months, however being below a threshold value). Considering distinction of higher quality, it is undesirable to classify a 10, 12 or 14-month aged ham as a 16-month ham, which happened in three cases (samples no. 14, 27, 30), or to classify 16 months old ham as less matured, which never occurred (nevertheless being close to that; namely a similar probability was obtained for two classes in the case of samples $57,61,62,51,60$ ).

Despite generally satisfactory results some weaknesses of the method must be mentioned. Firstly, some samples were classified into two or even more classes with a similar probability (uncertain). a) Model: $10 \times 10$ neurons, 100 epochs

\begin{tabular}{|c|c|c|c|c|c|}
\hline & \multicolumn{4}{|c|}{ True } \\
\hline & & 10 & 12 & 14 & 16 \\
\hline \multirow{5}{*}{$\begin{array}{l}\overrightarrow{0} \\
\frac{0}{0} \\
: 0 \\
0 \\
0\end{array}$} & 10 & 12 & 0 & 0 & 0 \\
\hline & 12 & 0 & 13 & 0 & 0 \\
\hline & 14 & 3 & 0 & 14 & 2 \\
\hline & 16 & 0 & 1 & 0 & 10 \\
\hline & Uncertain & 1 & 2 & 2 & 4 \\
\hline \multirow{2}{*}{\multicolumn{2}{|c|}{$\begin{array}{r}\text { Correct total } \\
\text { Uncertain total }\end{array}$}} & 49 & $76.6 \%$ & & \\
\hline & & 9 & $14.1 \%$ & & \\
\hline
\end{tabular}

b)Model: $10 \times 10$ neurons, 150 epochs

\begin{tabular}{|c|c|c|c|c|c|}
\hline & \multicolumn{4}{|c|}{ True } \\
\hline & & 10 & 12 & 14 & 16 \\
\hline \multirow{5}{*}{ 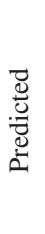 } & 10 & 12 & 0 & 1 & 0 \\
\hline & 12 & 0 & 12 & 0 & 0 \\
\hline & 14 & 3 & 0 & 14 & 1 \\
\hline & 16 & 1 & 1 & 0 & 11 \\
\hline & Uncertain & 0 & 3 & 1 & 4 \\
\hline \multirow{2}{*}{\multicolumn{2}{|c|}{$\begin{array}{r}\text { Correct total } \\
\text { Uncertain total }\end{array}$}} & 49 & $76.6 \%$ & & \\
\hline & & 8 & $12.5 \%$ & & \\
\hline
\end{tabular}

c) Model: $12 \times 12$ neurons, 200 epochs

\begin{tabular}{cc|cccc} 
& & \multicolumn{5}{|c}{ True } \\
& & 10 & 12 & 14 & 16 \\
\hline & 10 & 14 & 0 & 0 & 0 \\
& 12 & 0 & 11 & 0 & 0 \\
& 14 & 0 & 0 & 14 & 0 \\
\hline & 16 & 1 & 3 & 0 & 12 \\
\hline & Uncertain & 1 & 2 & 2 & 4 \\
\hline & Correct total & 51 & $79.7 \%$ & &
\end{tabular}

Fig. 2. Misclassification tables with the number of correct, false and uncertain predictions for the best three models.

In the case of representative samples (or good examples) all models produced the same results with high probability. In the case of untypical samples different models produce somewhat different results. To avoid this problem, different models for the detection of ham maturity could be used simultaneously in such cases. It is noteworthy that 12-months old hams which were misclassified were mainly placed into the class of 16-months. Similarly, the majority of uncertain 16-month hams (e.g. 57, 61, 62, 60) had similar probability for class12-months. Poor predictions could be related to the chemical composition of individual samples. It can be noted that dry-cured hams matured for 12 and 16 months had similar average water content (Table 1) which could explain uncertain distinction between these two classes. On the other hand, a lot of hams were correctly classified despite similar water content owing to the capability of NIR spectroscopy to detect other chemical (and physical) attributes. Misclassified or uncertainly predicted samples are often atypical representatives of a certain maturity class in relation to chemical composition. For example samples no. 14 

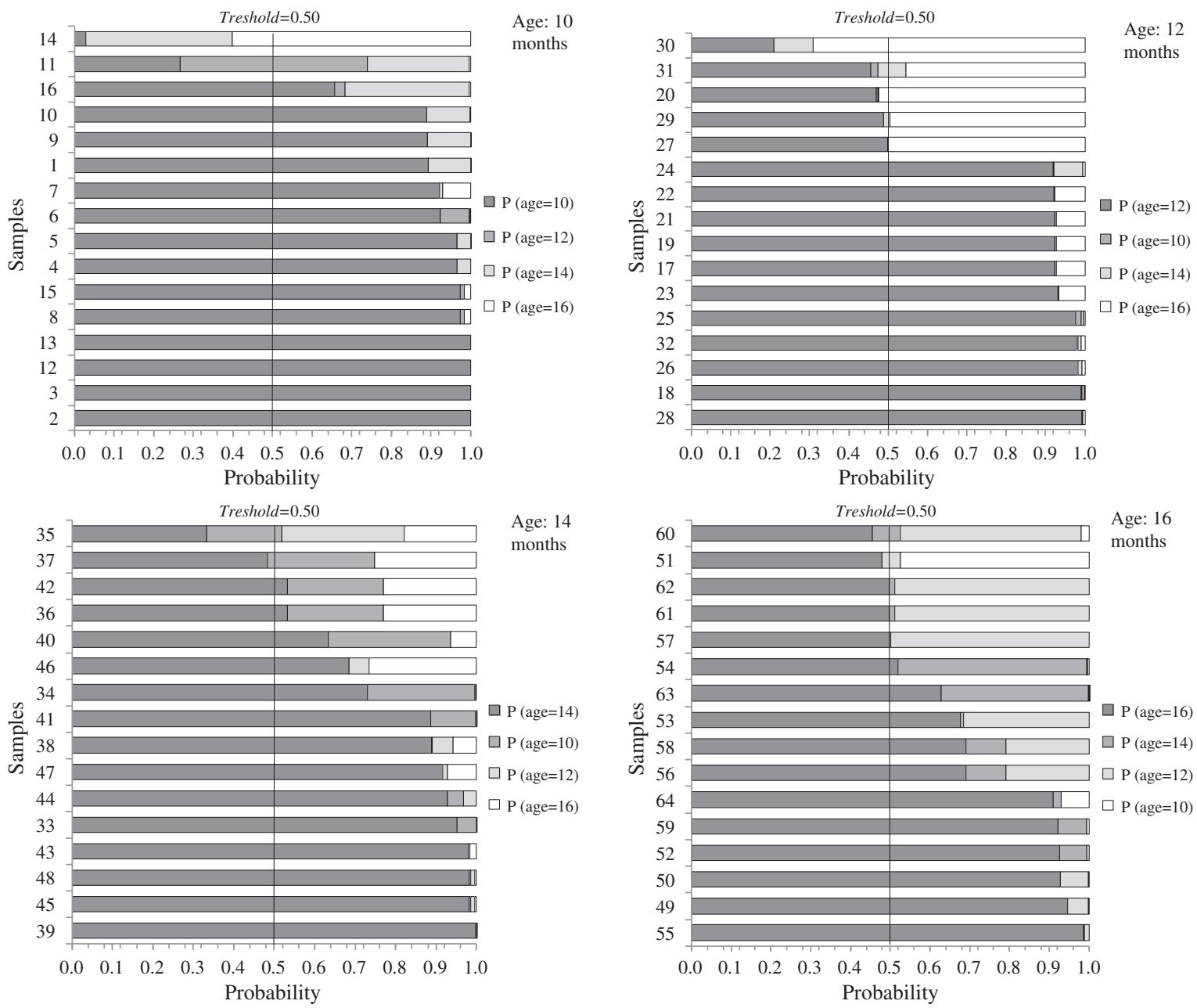

Fig. 3. Classification of ham samples according to the maturation time using the model with $12 \times 12$ neurons trained for 200 epochs based on NIR spectral information.

(10 months) and 30 (12 months) had very low water content which is probably the reason to be classified as 16 month matured hams. On the contrary, 16 month matured hams no. 61 and 62 had unusually high water content and were classified with similar probability $(\approx 0.5)$ in class 12 - and 16-months. Another example is the sample no. 35 (14 months) which had similar probability $(0.2-0.3)$ to belong to all four classes. This sample had an average water content with relatively low salt content which is characteristic for less matured hams and on the other hand a high proteolysis index (non-protein nitrogen content) known to increase with the maturity. The reason for uncertainty may be related to the differences in a dynamics of dehydration and salt intake and consequently other biochemical processes during the ripening which are affected by morphological or quality characteristics of the green hams (Čandek-Potokar \& Škrlep, 2012).

In the literature we found one scientific paper on the prediction of ham seasoning time reported by Lerma-García, Herrero-Martínez, Ramis-Ramos, Mongay-Fernández, and Simó-Alfonso (2009) who applied a capillary zone electrophoresis to predict curing time of Spanish hams using peptide profiles. However, no application of NIR spectroscopy for this purpose has been documented although the method was recently used for numerous different analyses on dry-cured hams (for review see Prieto et al., 2009). Moreover, several successful applications of NIR spectroscopy in a combination with ANN were reported in meat production and technology (a review of Prevolnik, Škorjanc, et al., 2011; Prevolnik, Škrlep, et al., 2011) and the results of the present study represent another promising example.

\section{Conclusion}

In the present study, a discrimination of dry-cured hams according to maturation time $(10,12,14,16$ months) based on NIR spectral information and application of ANN was performed. The accuracy of prediction (using a net $12 \times 12$ with 200 epoch) in external validation was $79.7 \%$, with $14.1 \%$ of uncertain predictions and no false positive ones. This result indicates the practical relevance of the studied approach. It should be noted that the results are valid for one type of the product (Kraški pršut). The potential for general use on different types of dry-cured hams needs verification.

\section{Acknowledgment}

The authors acknowledge the financial support from the state budget by the Slovenian Research Agency (programmes P4-0133, P4-0234, P1-0017, P1-0164). The support of COST Action FA 1102 FAIM is also greatly appreciated.

\section{References}

Benedini, R., Parolari, G., Toscani, T., \& Virgili, R. (2012). Sensory and texture properties of Italian typical dry-cured hams as related to maturation time and salt content. Meat Science, 90, 431-437.

Buscailhon, S., \& Monin, G. (1994). Déterminisme des qualités sensorielles du jambon sec. Chapitre 1: Evolution de la composition et des qualités sensorielles du jambon sec au cours de la fabrication. Viandes et Produits carnés, 15, 23-34. 
Čandek-Potokar, M., \& Škrlep, M. (2012). Factors in pig production that impact the quality of dry-cured ham: A review. Animal, 6, 327-338.

Cartwright, H. M. (2008). Using artificial intelligence in chemistry and biology: A practical guide. : Taylor \& Francis.

Commission implementing regulation (EU) no. 506/2012 of 14 June 2012 entering a name in the register of protected designations of origin and protected geographical indications (Kraški pršut (PGI)).

Dayhof, J. (1990). Neural network architectures, an introduction. New York: Van Nostrand Reinhold.

Gramatica, P., Pilutti, P., \& Papa, E. (2004). Validated QSAR prediction of $\mathrm{OH}$ tropospheric degradation of VOCs: Splitting into training-test sets and consensus modeling. Journal of Chemical Information and Computer Sciences, 44, 1794-1802.

Hecht-Nielsen, R. (1987). Counterpropagation networks. Appled Optics, 26, 4979-4984.

Lerma-García, M. J., Herrero-Martínez, J. M., Ramis-Ramos, G., Mongay-Fernández, C., \& Simó-Alfonso, E. F. (2009). Prediction of the curing time of Spanish hams using peptide profiles established by capillary zone electrophoresis. Food Chemistry, 113, 635-639.

Pérez-Marín, D., Garrido-Varo, A., \& Guerrero, J. E. (2007). Non-linear regression methods in NIRS quantitative analysis. Talanta, 72, 28-42.

Prevolnik, M., Čandek-Potokar, M., \& Škorjanc, D. (2004). Ability of NIR spectroscopy to predict meat chemical composition and quality - A review. Czech Journal of Animal Science, 49, 500-510.

Prevolnik, M., Škorjanc, D., Čandek-Potokar, M., \& Novič, M. (2011). Application of artificial neural networks in meat production and technology. In Kenji Suzuki (Ed.), Artificial neural networks - Industrial and control engineering applications (pp. 223-240). Rijeka: InTech. http://dx.doi.org/10.5772/15077 (http://www.intechopen.com/books/artificialneural-networks-industrial-and-control-engineering-applications/applicationof-artificial-neural-networks-in-meat-production-and-technology).
Prevolnik, M., Škrlep, M., Janeš, L., Velikonja-Bolta, Š., Škorjanc, D., \& Čandek-Potokar, M. (2011). Accuracy of near infrared spectroscopy for prediction of chemical composition, salt content and free amino acids in dry-cured ham. Meat Science, 88 299-304.

Prieto, N., Roehe, R., Lavín, P., Batten, G., \& Andrés, S. (2009). Application of near infrared reflectance spectroscopy to predict meat and meat products quality: A review. Meat Science, 83, 175-186.

Roncaglioni, A., Novič, M., Vračko, M. \& Benfenati, E (2004). Classification of potential endocrine disrupters on the basis of molecular structure using a nonlinear modeling method. Journal of Chemical Information and Computer Sciences, 44 300-309.

Škrlep, M., Čandek-Potokar, M., Žlender, B., Robert, N., Santé-Lhoutellier, V., \& Gou, P. (2012). PRKAG3 and CAST genetic polymorphisms and quality traits of dry-cured hams-III. Associations in Slovenian dry-cured ham Kraški pršut and their dependence on processing Original Research Article. Meat Science, 92(4), 360-365.

Toldra, F., \& Flores, M. (1998). The role of muscle proteases and lipases in flavour development during the processing of dry-cured ham. Critical Reviews in Food Science, $38,331-352$.

Virgili, R., Saccani, G., Gabba, L., Tanzi, E., \& Soresi Bordini, C. (2007). Changes of free amino acids and biogenic amines during extended ageing of Italian dry-cured ham. LWT-Food Science and Technology, 40, 871-878.

Zupan, J. (1994). Introduction to artificial neural network (ANN) methods: What they are and how to use them. Acta Chimica Slovenica, 41, 327-352.

Zupan, J., Novič, M., \& Ruisanchez, I. (1997). Kohonen and counterpropagation artificia neural networks in analytical chemistry. Chemometrics and Intelligent Laboratory Systems, 38, 1-23. 